H. HOU, X. SHEN, C. WANG, L. ZHANG, P. RÖSE, L.-A. CHEN, K. HARMS, M. MARSCH, G. HILT, E. MEGGERS* (PHILIPPS-UNIVERSITÄT MARBURG, GERMANY AND XIAMEN UNIVERSITY, P. R. OF CHINA)

Asymmetric Photoredox Transition-Metal Catalysis Activated by Visible Light Nature 2015, 515, 100-103.

\section{Asymmetric Alkylation via Photoredox Pathway Using a Chiral Iridium Complex}

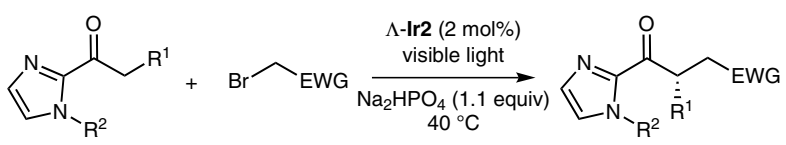

Selected examples:<smiles></smiles>
$1.5 \mathrm{~h}, 100 \%$ yield, $99 \%$ ee<smiles>O=C(c1ncc[nH]1)C1Cc2c(cc([N+](=O)[O-])cc2[N+](=O)[O-])-c2ccccc21</smiles>
$2 \mathrm{~h}, 93 \%$ yield, $99 \%$ ee<smiles>CC(Cc1ccc([N+](=O)[O-])cc1C#N)C(=O)c1ncc[nH]1</smiles>

$24 \mathrm{~h}, 87 \%$ yield, $97 \%$ ee

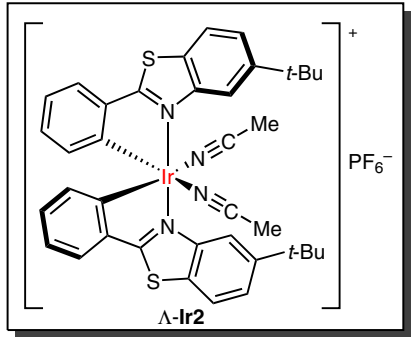<smiles></smiles>

$2 \mathrm{~h}, 98 \%$ yield, $99 \%$ ee<smiles></smiles><smiles>CC(Cc1ccc([N+](=O)[O-])cc1[N+](=O)[O-])C(=O)c1ncc[nH]1</smiles>

$36 \mathrm{~h}, 84 \%$ yield, $91 \%$ ee<smiles></smiles>

$6 \mathrm{~h}, 97 \%$ yield, $94 \%$ ee<smiles>O=C(c1ncc[nH]1)[C@H](Cc1ccc([N+](=O)[O-])cc1[N+](=O)[O-])c1ccsc1</smiles>

$2 \mathrm{~h}, 99 \%$ yield, $97 \%$ ee<smiles></smiles>

Proposed mechanism:
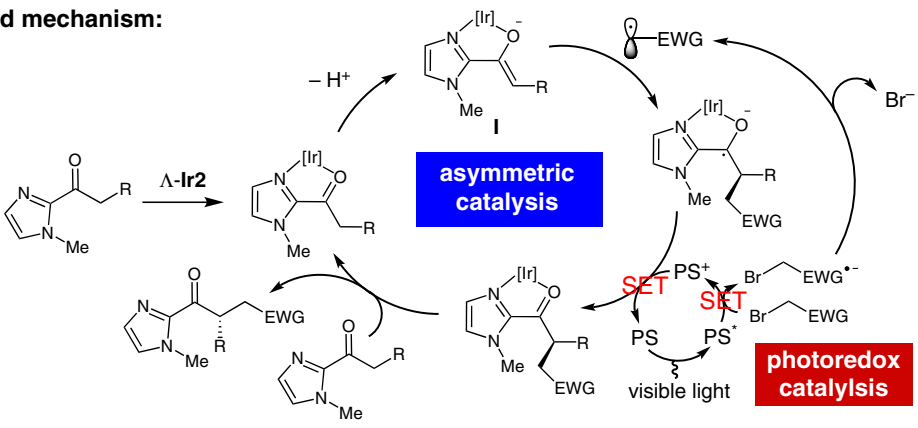

EWG: electron-withdrawing group

SET: single electron transfer

PS: photosensitizer in the form of enolate complex I

Significance: The authors demonstrate a unique example of asymmetric redox catalysis achieved by visible light. Two catalytic cycles are connected by the key intermediate, an iridium(III) enolate complex, which is an active photosensitizer generated in situ.
Comment: This process provides very mild conditions and excellent yields and enantioselectivities. The reaction proceeds via a photoredox mechanism, supported by X-ray, radical trapping, luminescence quenching, and interval experiment analyses.

SYNFACTS Contributors: Hisashi Yamamoto, Takayuki Furukawa

Dol: 10.1055/s-0034-1379825; Reg-No.: H16614SF 